\title{
Demanda de gasolina en la zona metropolitana del Valle de México: análisis empírico de la reducción del subsidio
}

Recibido: Junio, 2013 - Aceptado: Febrero, 2014

Doi: dx.doi.org/10.12804/rev.econ.rosario.17.01.2014.04

\author{
Jimy Ferrer Carbonell ${ }^{*}$ \\ Comisión Económica para América Latina y el Caribe
}

Roberto Escalante Semerena ${ }^{\dagger}$

Universidad Nacional Autónoma de México

\section{Resumen}

Este artículo analiza la demanda de gasolina en la Zona Metropolitana del Valle de México (ZMVM). Se modela el consumo de gasolina como una función del ingreso y el precio relativo. Utilizando el enfoque de cointegración se encuentra una relación estable y de largo plazo entre las variables, que indica que la respuesta de la demanda es mayor ante cambios en el ingreso que frente a cambios en el precio, por lo que las políticas públicas basadas solo en precios no son suficientes para disminuir el consumo de gasolina en esta zona metropolitana, sino que estas deben complementarse con medidas de regulación.

Clasificación JEL: Q41, R41, H23, Q48, C22.

Palabras clave: elasticidad demanda de gasolina, subsidios, política pública, cointegración.

* Oficial de Asuntos Económicos, Comisión Económica para América Latina y el Caribe. Av. Dag Hammarskjöld 3477. Vitacura. Santiago. Chile. Teléfono: (56 2) 2210 2756. Fax: (56 2) 208 0484. Correo electrónico: jimy.ferrer@cepal.org - jimyferrerc@yahoo.com.co

† Profesor e investigador de la Facultad de Economía, Universidad Nacional Autónoma de México, UNAM. Ciudad de México. Correo electrónico: semerena@unam.mx

Para citar este artículo: Ferrer, C. J., \& Escalante, S. R. (2014). Demanda de gasolina en la zona metropolitana del Valle de México: análisis empírico de la reducción del subsidio. Revista de Economía del Rosario, 17(1), 89-117. doi: dx.doi.org/10.12804/rev.econ.rosario.17.01.2014.04 


\title{
Gasoline Demand in Mexico City Metropolitan Area: An empirical Analysis of Reduction in Subsidy
}

\begin{abstract}
This article analyses the demand of gasoline in the Mexico City Metropolitan Area. The gasoline consumption is modeled as a function of income and relative prices. Using the cointegration approach, we obtain a stable and long term relationship between the variables, which indicate that the response of the demand is higher to changes in income than to changes in prices, which means, that public policies based on prices only are not sufficient to reduce the consumption of gasoline in this metropolitan area, but rather these must be complemented by regulation measures.
\end{abstract}

JEL Classification: Q41, R41, H23, Q48, C22.

Keywords: Gasoline demand elasticities, subsidies, public policy, cointegration.

\section{Demanda de gasolina na zona metropolitana do Vale do México: Análise empírica da redução do subsídio}

\author{
Resumo
}

Este artigo analisa a demanda de gasolina na Zona Metropolitana do Vale do México (ZMVM). Modela-se o consumo de gasolina como uma função de ingresso e o preço relativo. Empregando o enfoque de cointegração encontra-se uma relação estável e de longo prazo entre as variáveis, que indica que a resposta da demanda é maior ante mudanças no ingresso que frente a mudanças no preço, pelo que políticas públicas baseadas só em preços não são suficientes para diminuir o consumo de gasolina nesta zona metropolitana, senão que estas devem se complementar com medidas de regulação.

Classificação JEL: Q41, R41, H23, Q48, C22.

Palavras-chave: elasticidade, demanda de gasolina, subsídios, política pública, cointegração. 


\section{Introducción}

En las últimas décadas, el estudio de la demanda de gasolina ha tenido mayor importancia en el ámbito internacional, entre otros motivos, por la escasez y el aumento del precio del petróleo, las emisiones de gases de efecto invernadero (GEI) y otros efectos ambientales asociados a su consumo y las implicaciones sobre las finanzas públicas. En países donde se subsidia la gasolina y en zonas altamente pobladas, donde el parque vehicular suele crecer a un ritmo acelerado, el estudio de la demanda de gasolina resulta particularmente importante por la utilidad que tiene para la formulación de políticas públicas. En el caso de México los precios de la gasolina y el diesel son administrados, por consiguiente, no responden a las leyes del mercado sino que son fijados por la Secretaría de Hacienda y Crédito Público (SHCP) tomando como base los precios del productor en el mercado internacional (Reyes, 2011). Esta estructura de precios permite que en México exista un subsidio a la gasolina y el diesel cuando el precio que pagan los consumidores finales es menor que el precio que se paga en los Estados Unidos (SENER, 2010). No obstante, las características de las finanzas públicas en México, los compromisos en materia ambiental, por ser país miembro de la OCDE, y sus objetivos de reducir las emisiones de GEI, declarados en el marco de la Convención Marco de las Naciones Unidas sobre Cambio Climático, entre otros factores, motivaron una reforma tributaria que incluye la reducción de los subsidios a la gasolina.

En este contexto, el objetivo de este artículo es estimar una función de demanda de gasolina para la Zona Metropolitana del Valle de México por medio de la técnica de cointegración y encontrar las elasticidades precio e ingreso. A partir de los resultados que se obtienen, se construyen escenarios de reducción del subsidio que permitirán realizar proyecciones para el consumo de gasolina y presentar recomendaciones de política pública.

El artículo está estructurado en siete secciones. La primera sección es esta introducción. En la segunda se presenta el estado del arte sobre la demanda de gasolina; en la tercera se presentan los fundamentos teóricos detrás de la demanda de gasolina; en la cuarta se describen la técnica de cointegración y el modelo econométrico utilizado; $y$ en la quinta se dan a conocer las elasticidades de largo y corto plazo que se obtienen para la Zona Metropolitana del Valle de México. Seguidamente, en la sexta sección se construyen escenarios que permiten realizar simulaciones para el consumo de gasolina hasta $2020 \mathrm{y}$, finalmente, en la séptima sección, se dan a conocer algunas consideraciones finales e implicaciones de política pública. 


\section{Revisión de literatura}

En el ámbito internacional son muchos los trabajos que se han realizado sobre la demanda de gasolina para transporte por carretera. Gran parte de estos estudios han estado motivados por la preocupación mundial ante la escasez de petróleo $y$, en el caso de las grandes ciudades, por los efectos adversos que tiene el crecimiento del consumo de gasolina sobre el medio ambiente y la salud. La literatura internacional sobre la demanda de gasolina muestra diferentes resultados. Esto es, las magnitudes de las elasticidades precio e ingreso de la demanda de gasolina varían entre regiones, por una parte por las particularidades del mercado de combustibles en cada región, pero también por aspectos metodológicos como la especificación de los modelos, las formas funcionales, horizonte de tiempo considerado, metodología econométrica utilizada y el tipo y periodicidad de los datos (Espey, 1998; Dahl y Sterner, 1991; Graham y Glaister, 2002).

Algunos trabajos se han interesado en estimar la demanda de gasolina por medio del uso de microdatos (Archibald y Gillingham, 1980; Kayser, 2000; Mannering y Winston, 1985). La estimación de la demanda de gasolina a partir de microdatos en lugar de datos agregados ha sido justificada porque proporciona estimaciones que reflejan mejor la respuesta de los consumidores ante cambios en los precios de la gasolina o en el ingreso familiar, lo que permite ver al mismo tiempo cómo las características específicas de un hogar afectan la demanda de gasolina. En este sentido, al utilizar mínimos cuadrados en dos etapas se encontró para el caso de Estados Unidos que la elasticidad precio es -0,23 y la elasticidad ingreso de 0,49 (Kayser, 2000).

De igual manera, se ha intentado medir las diferencias entre las elasticidades de la demanda de gasolina en diferentes periodos donde los precios de la gasolina presentaron un comportamiento similar en Estados Unidos. Utilizando mínimos cuadrados ordinarios, con datos de 2001 a 2006, se encontró que la elasticidad precio de corto plazo está en un rango de $-0,03$ a $-0,07$, menor que la elasticidad precio de -0,21 a -0,34 obtenida para el periodo 1975-1980 (Hughes, et al., 2006).

Por otra parte, se encuentran estudios de demanda de gasolina que han utilizado datos panel para estimar las elasticidades precio e ingreso. Por medio de un panel no balanceado conformado por noventa países (de diferentes niveles de ingreso) para el periodo 1990-1997 se encontró que la omisión de las características de distribución del ingreso proporciona elasticidades engañosas (Storchmann, 2005), concluyéndose que en los países pobres una distribución desigual del ingreso es necesaria para que al menos algunas personas puedan comprar automóviles, mientras que en los países ricos una distribución desigual del ingreso excluye a algunas personas de la adquisición de un automóvil. 
De igual manera, se pudo establecer que, independientemente de los modelos utilizados (mínimos cuadrados ordinarios o mínimos cuadrados generalizados), la elasticidad ingreso oscila entre 1,0 y 1,2 , mientras que la elasticidad precio está entre -0,8 y -1,0 (Storchmann, 2005).

Al estudiar la demanda de gasolina y de vehículos en Marruecos para el periodo 1970-1996, al utilizar un sistema de ecuaciones simultáneas, se encontró una baja elasticidad de corto plazo respecto del precio $(-0,13)$ e ingreso $(0,22)$, al igual que en el largo plazo, donde las elasticidades precio e ingreso de la demanda de gasolina fueron -0,3 y 0,5, respectivamente (Belhaj, 2002). El análisis de la demanda de gasolina en los países petroleros (Kuwait, Arabia Saudita, Qatar, UAE, Omán y Bahréin) mediante datos panel, para el periodo 1975-1993, muestra que la demanda de gasolina es inelástica respecto del precio $(-0,17)$ e ingreso $(0,48)$ en el corto plazo, al igual que en el largo plazo, donde se estimaron elasticidades de -0,11 y 0,31, concluyéndose que el crecimiento de la demanda de gasolina en estos países se atribuye al aumento en el ingreso y a la caída de los precios relativos (Eltony, 1996).

Otro grupo de investigadores ha estudiado la demanda de gasolina mediante el enfoque de cointegración (Amengual y Cubas, 2002; Akinboade, et al., 2008; Eltony y AI-Mutairi, 1995). Al usar la técnica de cointegración y un modelo de corrección de errores (MCE), para el periodo 1970-1989, se encontró que en Kuwait la demanda de gasolina es inelástica respecto del precio en el corto plazo $(-0,37)$ y en el largo plazo $(-0,46)$, mientras que respecto del ingreso la demanda es elástica en el largo plazo $(0,92)$ e inelástica en el corto plazo $(0,47)$, lo cual indica que la respuesta de la demanda de gasolina a los cambios en el ingreso es mayor en el largo plazo que en el corto plazo (Eltony y AI-Mutairi, 1995).

En América Latina una referencia importante en el estudio de la demanda de gasolina es el caso del Brasil donde se cuenta con un sustituto para la gasolina como el alcohol. La estimación de la elasticidad precio cruzada entre la gasolina y el alcohol, utilizando análisis de cointegración, permite determinar que el alcohol es un sustituto imperfecto de la gasolina, incluso en el largo plazo, lo que puede explicarse por los altos costos asociados con el cambio de motores de los automóviles a gasolina para el de combustible a base de alcohol (Alves y Bueno, 2003).

En un estudio para México, se estimó la elasticidad de la demanda de gasolina en el ámbito nacional en relación con el precio, el ingreso y el volumen de vehículos. Los resultados que se obtienen mediante un modelo de cointegración para el periodo 1980-2006 muestran que la demanda es inelástica respecto del precio $(-0,29)$, del ingreso $(0,53)$ y del stock de vehículos $(0,60)$, mientras que los resultados hallados utilizando datos panel para las 32 entidades federativas de la República mexicana, para el periodo 1993-2004, indican que la demanda de 
gasolina es elástica en relación con el ingreso $(1,19)$ y el volumen de vehículos $(1,58)$, e inelástica respecto del precio (-0,39) (Crotte, 2008).

Para el caso de la ZMVM se ha encontrado una alta elasticidad de largo plazo respecto del ingreso $(1,31)$ y una muy baja elasticidad respecto del precio $(-0,04)$, los autores concluyen que la elasticidad precio es muy baja como resultado de la exclusión de bienes sustitutos y que la elasticidad ingreso incide en el consumo, en gran medida, mediante la composición y crecimiento de la flota vehicular (Galindo y Salinas, 1997).

A partir de una extensa revisión de literatura sobre la demanda de gasolina, en la que se recopilaron 180 estimaciones que cubren el periodo 1960-2010, se realizaron histogramas que sintetizan las magnitudes de las elasticidades ingreso y precio de largo y corto plazo reportados en la literatura internacional. Como se ve en la figura 1, existe evidencia que muestra que la elasticidad ingreso de largo plazo de la demanda de gasolina está entre 0,5 y 0,7, mientras que la elasticidad precio está entre $-0,15$ y $-0,45$.

Elasticidad ingreso

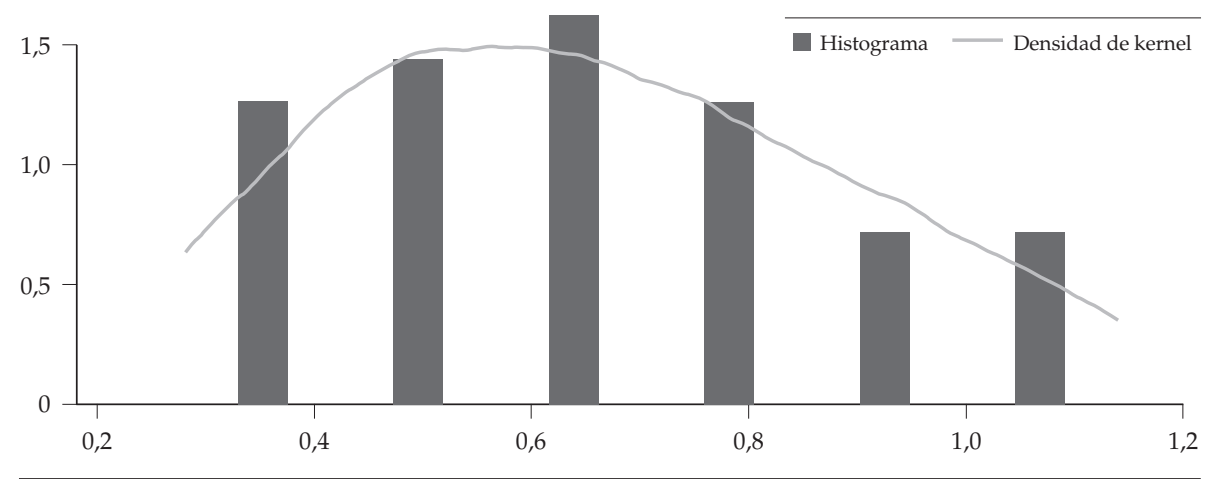

Elasticidad precio

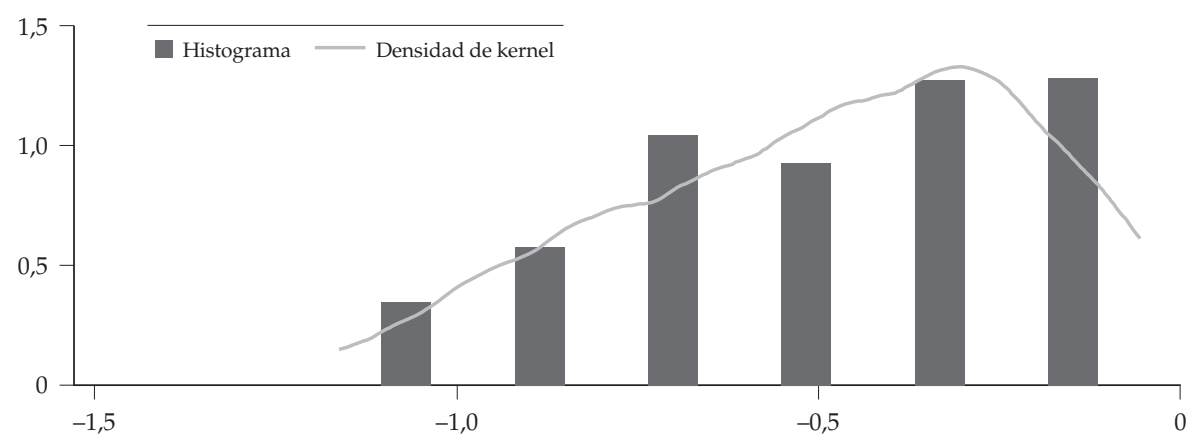

Figura 1. Distribución de las elasticidades de largo plazo de la demanda de gasolina Fuente: Elaboración propia a partir de la revisión de literatura. 
Como se ve en la figura 2, la revisión de la literatura de las elasticidades de corto plazo de la demanda de gasolina, resumida en los histogramas, indica que la elasticidad ingreso de corto plazo está entre 0,2 y 0,5, mientras que la elasticidad precio se ubica entre $-0,1$ y $-0,2$. Estos resultados muestran que la respuesta de los consumidores ante variaciones en el precio y el ingreso es menor en el corto plazo en comparación con la respuesta que se presenta en el largo plazo.

Elasticidad ingreso

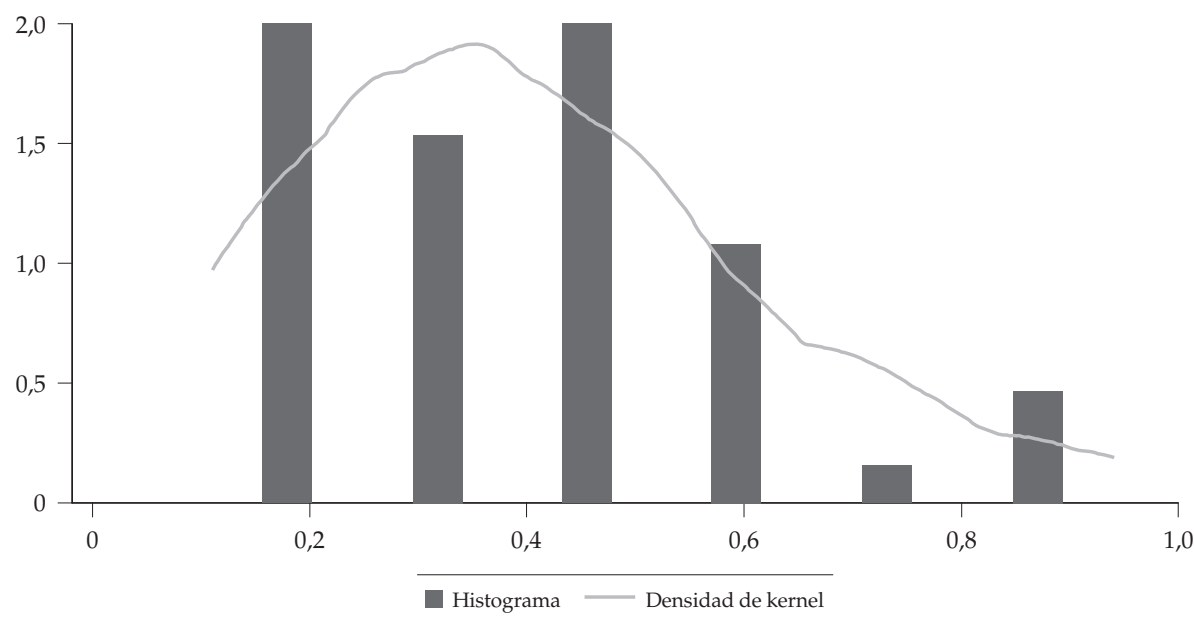

Elasticidad precio

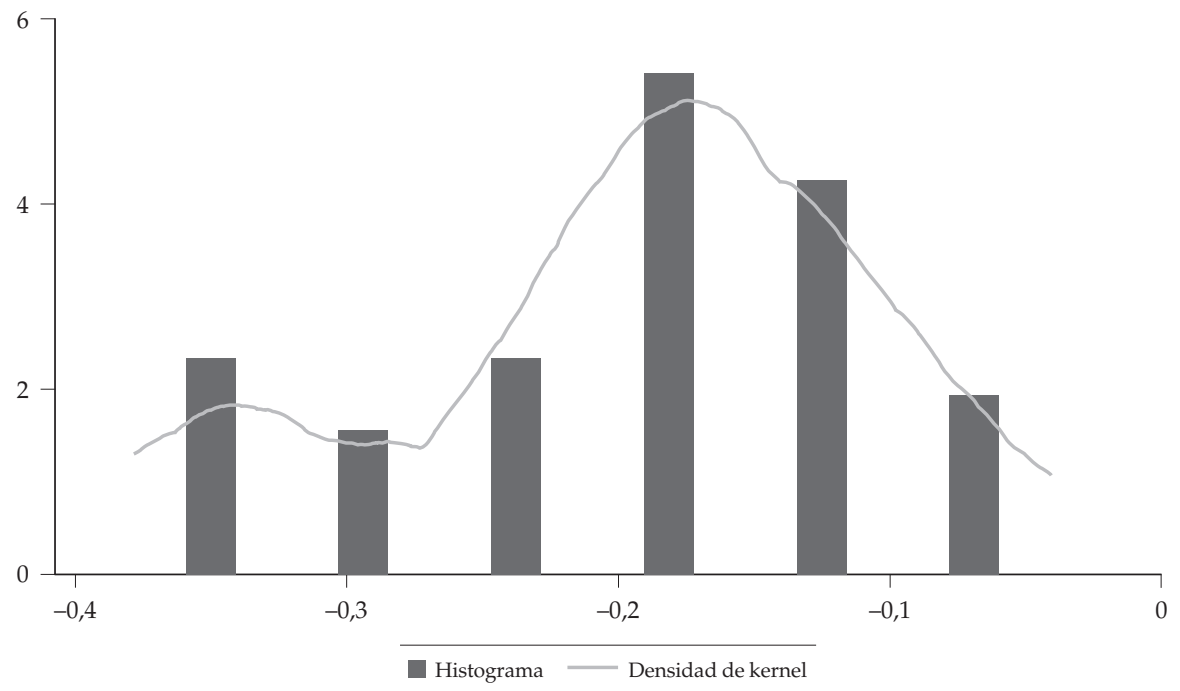

Figura 2. Distribución de las elasticidades de corto plazo de la demanda de gasolina Fuente: Elaboración propia a partir de la revisión de literatura. 


\section{Teoría de la demanda de gasolina}

De acuerdo con el enfoque de la teoría del consumidor, la demanda de gasolina es una demanda derivada (Becker, 1965; Lancaster, 1966; Muth, 1966). Esto es, no es el consumo de gasolina lo que le representa beneficios al consumidor, sino el producto final que resulta: movilidad, que es producida con la participación de varios inputs como vehículo, gasolina y tiempo. En este sentido, la demanda de combustible está determinada por una serie de decisiones de los consumidores: en primer lugar, deben decidir si compran o no un auto, lo cual está determinado por el stock de vehículos en la economía; en segundo lugar, el tipo de vehículo que decide comprar, lo cual a su vez determina la eficiencia del combustible; $y$, en tercer lugar está la decisión de cuánto utilizar el vehículo, que determina el kilometraje anual del stock de vehículos (Storchmann, 2005).

A pesar de que la demanda de gasolina puede ser considerada como una demanda derivada, esta puede ser modelada como cualquier otro bien, bajo el supuesto de separabilidad débil ${ }^{1}$ (Galindo y Salinas, 1997; Deaton y Muellbauer, 1980; Mora, 2002). En tal sentido, se puede analizar la demanda de gasolina bajo el marco de la teoría económica convencional, es decir, que teniendo en cuenta las preferencias de los individuos y dada la restricción presupuestaria que enfrentan, estos deciden adquirir una cesta de consumo que maximiza su utilidad (Varian, 1990; Mas-Colell, et al., 1995; Pincyck y Rubinfeld, 1998). De esta manera, gran parte de la literatura internacional sobre la demanda de combustibles resume la demanda de gasolina en función de su precio y del ingreso (Amengual y Cubas, 2002; Eltony y AI-Mutairi, 1995; Birol y Guerer, 1993; Bentzen, 1994; Ramanathan, 1999; Galindo, 2005, 2008).

En los últimos años, y en la medida que la disponibilidad de información lo permite, se han adicionado otras variables para explicar la demanda de gasolina como el stock de vehículos y la tarifa del transporte público (Crôtte, et al., 2010), el precio de sustitutos como el diesel y el alcohol y el número de vehículos

1 Según la separabilidad débil, la función de utilidad puede escribirse como $U=v\left(q_{1}\right.$, $\left.q_{2}, q_{3}, q_{4}, q_{5}, q_{6^{\prime}}\right)=f\left(V_{F}\left(q_{1}, q_{2}\right), V_{S}\left(q_{3}, q_{4}\right) V_{E}\left(q_{5}, q_{6}\right)\right)$ donde $f(\cdot)$ es una función creciente y $\left(V_{F}, V_{S}\right.$ y $\left.V_{E}\right)$ son funciones de subutilidad asociadas a grupos de bienes de consumo $\left(q_{1}, \ldots, q_{6}\right)$. La maximización de la utilidad $(U)$ implica que $\left(V_{F}, V_{S}\right.$ y $\left.V_{E}\right)$ son cada una maximizada sujeta a la restricción de cuanto se gasta en los grupos de bienes de consumo; si esto no fuera así, $\left(V_{F}\right.$, $V_{S}$ y $V_{E}$ ) podrían aumentar sin violar la restricción presupuestaria. Por consiguiente los gastos en $\left(q_{1}\right.$ y $\left.q_{2}\right)$ son el resultado de maximizar $V_{F}\left(q_{1}, q_{2}\right)$ sujeto a $p_{1} q_{1}+p_{2} q_{2}=x_{F}$ el gasto total en este grupo de bienes. De esta manera, la demanda marshaliana de cualquier grupo de bienes de consumo $\left(q_{i}\right)$ se puede escribir como: $q_{1}=g_{F i}\left(x_{F}, p_{1}, p_{2}\right), i=1,2$. Esta función de demanda posee todas las propiedades usuales de una función de demanda dado que ellas derivan de un problema de maximización de utilidad estándar (Una completa explicación de la separabilidad débil se encuentra en Deaton y Muelbauer (1980). 
por persona (Eltony, 1996; Alves y Bueno, 2003; Polemis, 2006). Las necesidades de buscar una reducción en el consumo de gasolina en regiones altamente pobladas y con gran volumen de vehículos han llevado también a estudiar los efectos de una mejora de la eficiencia de los vehículos utilizados, medido como los kilómetros por litro o la intensidad de la gasolina, medida como consumo por vehículo, mientras que otros trabajos acerca de este tema en la literatura internacional han incluido el precio de los automóviles, la venta y la malla vial como variables explicativas (Baltagi y Griffin, 1983; Eskeland y Feyzioglu, 1997; Galindo, 2008; Johansson y Schipper, 1997; Storchmann, 2005).

En cuanto a la forma funcional, con la cual se ha modelado la demanda de gasolina, el patrón dominante ha sido utilizar una función lineal doble logarítmica (Akinboade, et al., 2008). La ventaja de utilizar esta forma radica en la facilidad para interpretar sus resultados. Los coeficientes que se obtienen de estas regresiones se pueden interpretar de manera directa como elasticidades que miden la sensibilidad con la que cambia la variable dependiente frente a cambios en las variables explicativas.

\section{Modelación econométrica de la demanda de gasolina}

La literatura internacional reporta amplia evidencia que muestra al ingreso y al precio como variables determinantes del consumo de gasolina (Storchmann, 2005; Bentzen, 1994; Crôtte, et al., 2010; Dahl y Sterner, 1990; Sterner, et al., 1992; Johnston \& DiNardo, 1997; Sultan, 2010); sin embargo, estas variables muestran una clara tendencia que pueden ocasionar el problema de regresión espuria (Reyes, 2010), por lo que no se recomienda la aplicación de técnicas econométricas tradicionales. ${ }^{2}$ Estas series presentan un proceso estocástico no estacionario, por consiguiente solo podrán ser utilizadas en un modelo econométrico si existe una combinación lineal entre ellas que genere un proceso estocástico estacionario.

La especificación de modelos de demanda de gasolina más utilizada corresponde a una función lineal donde todas las variables están expresadas en logaritmos naturales (Eltony, 1996; Akinboade, et al., 2008; Galindo, 2008; Dahl \& Sterner, 1990; Reyes, 2010), como se especificada en la ecuación (1).

2 La econometría moderna ha encontrado consenso en que el enfoque de cointegración ofrece ventajas en el tratamiento de los datos que pueden mostrar alguna tendencia. En el apéndice metodológico se presenta en detalle la técnica de cointegración. 


$$
L G_{t}=\beta_{0}+\beta_{1} L Y_{t}+\beta_{2} L P_{t}+\sum_{i=1}^{n} \beta_{1} X_{t}+\varepsilon_{t}
$$

Donde $G$ representa el consumo de gasolina, $Y$ es el ingreso y $P$ el precio del combustible. Por su parte, $X$ representa otras variables que pueden explicar la demanda de gasolina como el stock de autos, la venta, el precio, entre otras. De acuerdo con la teoría económica, $\beta_{1}$ representa la elasticidad ingreso y $\beta_{2}$ la elasticidad precio de la demanda, esperándose que $\beta_{1}$ sea positivo y $\beta_{2}$ negativo.

\section{Evidencia empírica}

Siguiendo los trabajos de (Akinboade, et al., 2008; Alves y Bueno, 2003; Eltony, 1996; Galindo, 2008; Polemis, 2006; Ramanathan, 1999) se especificó el modelo de la demanda de gasolina con una forma funcional log-lineal, de la siguiente manera:

$$
c g_{t}=\beta_{0}+\beta_{1} y_{t}+\beta_{2} \operatorname{prg}_{t}+\varepsilon_{t}
$$

Todas las variables del modelo están en logaritmos y sus valores corresponden a observaciones anuales del periodo 1980-2012 para la ZMVM. La variable dependiente $c g$ es el consumo de gasolina (miles de litros anuales). La variable explicativa $y$ es el nivel de ingreso (PIB en millones de pesos de 1993), prg es el precio relativo del combustible (calculado como el índice general de precios de las gasolina entre el índice de precios al consumidor), mientras que $\varepsilon$ es un error aleatorio que, se supone, es ruido blanco y normal e idénticamente distribuido.

La dinámica de las series utilizadas en el modelo se presenta en la figura 3. Como se puede observar, existe una tendencia creciente en el comportamiento de estas variables, lo que puede llevar a pensar que el aumento del consumo de gasolina en la ZMVM ha estado relacionado con el crecimiento que ha registrado el ingreso y el estancamiento del precio de la gasolina.

Para las series consumo de combustible, ingreso, precio relativo de la gasolina y venta de autos, se realizaron las pruebas Dickey-Fuller Aumentada (ADF), Phillips y Perron (PP) y Kwiatkowsky, Phillips, Schmidt y Shin (KPSS) para conocer su orden de integración, utilizando el software E-views 6.0. Los resultados de las pruebas se presentan en la tabla 1, donde se puede ver que todas las series son no estacionarias de orden de integración I(1). 
Consumo de gasolina (Millones de litros)

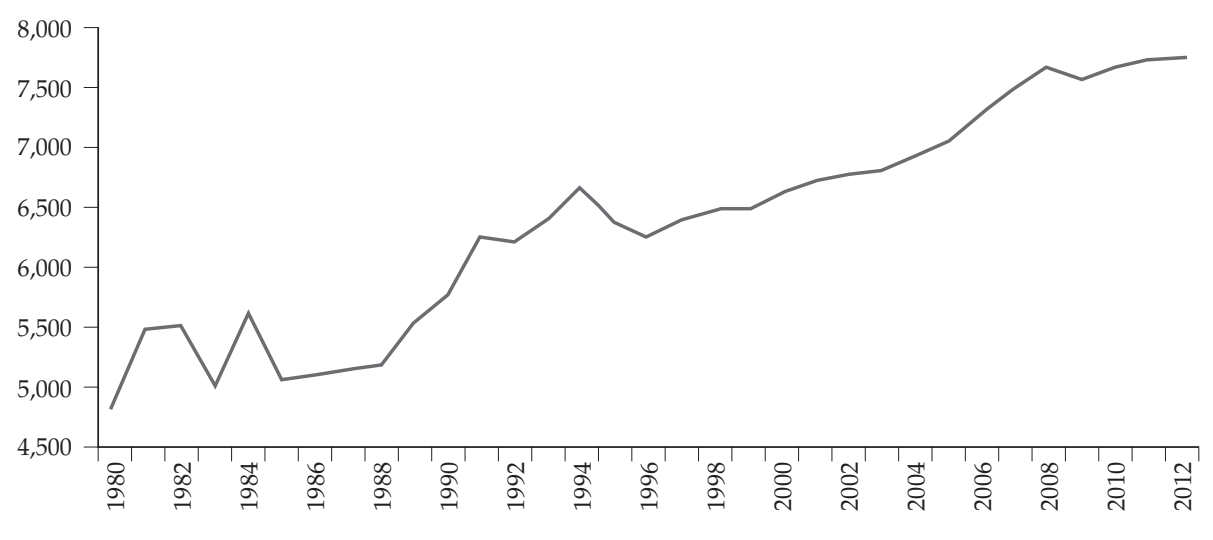

PIB (Miles de pesos de 1993)

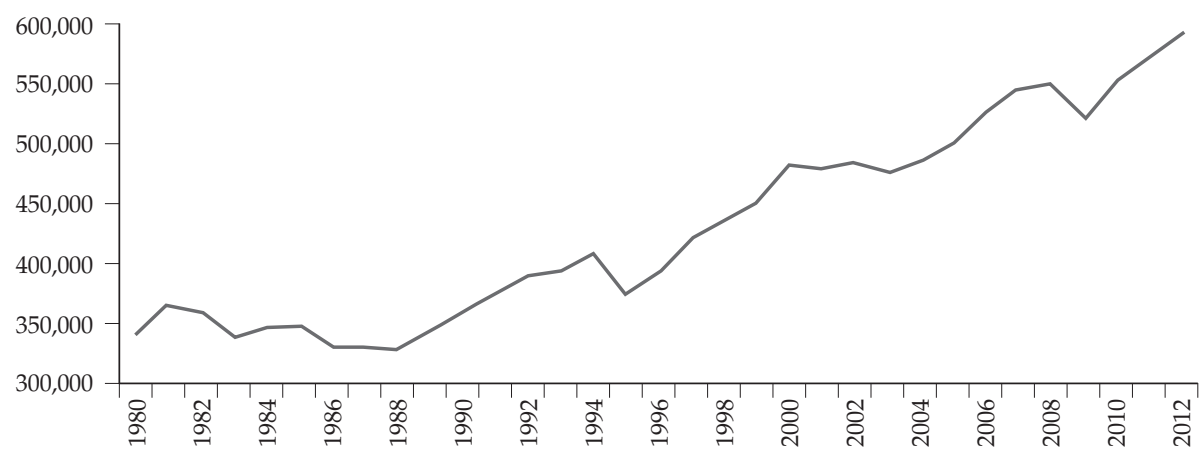

Precios relativos de la gasolina

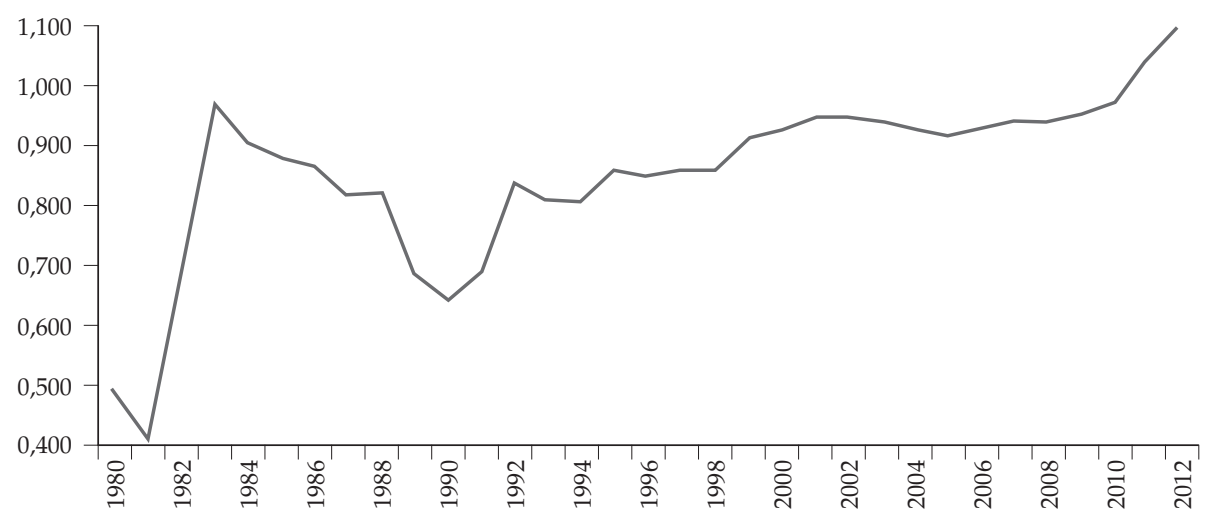

Figura 3. Comportamiento de variables asociadas al consumo de gasolina en la ZMVM 1980-2012

Fuente: Elaboración propia a partir de datos del INEGI, PEMEX, SENER y Banco de México. 
Tabla 1. Pruebas de raíz unitaria sobre estacionariedad

\begin{tabular}{|c|c|c|c|c|c|c|c|c|}
\hline Prueba & & $\mathrm{ADF}$ & & & PP (3) & & & \\
\hline Serie & A & B & C & A & B & C & $\eta_{\mu}$ & $\eta_{\tau}$ \\
\hline $\operatorname{lcg}$ & $-3,304(7)$ & $-1,309(0)$ & $1,796(0)$ & $-3,650$ & $-1,264$ & 2,231 & 0,568 & 0,070 \\
\hline$\Delta \mathrm{lcg}$ & $-3,638(7)$ & $-8,381(0)$ & $-7,707(0)$ & $-8,076$ & $-8,215$ & $-7,357$ & 0,115 & 0,081 \\
\hline ly & $-2,297(0)$ & $0,270(0)$ & $2,509(0)$ & $-2,202$ & 0,383 & 2,656 & 0,559 & 0,129 \\
\hline$\Delta \mathrm{ly}$ & $-5,576(0)$ & $-5,294(0)$ & $-4,723(0)$ & $-5,596$ & $-5,294$ & $-4,774$ & 0,239 & 0,114 \\
\hline lprg & $-3,581(4)$ & $-2,827(0)$ & $-2,888(0)$ & $-3,134$ & $-2,796$ & $-1,948$ & 0,579 & 0,087 \\
\hline$\Delta \mathrm{lprg}$ & $-1,517(8)$ & $-1,611(8)$ & $-0,148(8)$ & $-5,248$ & $-5,114$ & $-4,922$ & 0,142 & 0,101 \\
\hline
\end{tabular}

Notas: Los valores en negritas representan el rechazo de la hipótesis nula. Los valores críticos al $5 \%$ de significancia para las pruebas Dickey-Fuller Aumentada (ADF) y Phillips-Perron (PP) tomando una muestra $\mathrm{T}=100$ son: Modelo $\mathrm{A}=-3,45$ (incluye constante y tendencia), Modelo $\mathrm{B}=-2,89$ (incluye constante) y Modelo C $=-1,95$ (no incluye constante y tendencia).

Fuente: Maddala \& Kim (1998), p. 64. Los valores críticos de la prueba KPSS a un nivel del $5 \%$ de significancia son: $\eta_{\mu}=0,463$ (incluye constante) y $\eta_{\tau}=0,146$ (incluye constante y tendencia). Kwiatkowski, Phillips, Schmidt \& Shin (1992). Periodo 1980-2012.

A pesar del extenso uso de las pruebas ADF, PP y KPSS en los trabajos aplicados, la presencia de cambios estructurales en las series puede llevar a que estas pierdan validez. Esto es, los cambios estructurales en las series pueden ser identificados como la presencia de raíz unitaria, dificultando la distinción entre series estacionarias con cambio estructural de aquellas con raíces unitarias genuinas (Maddala \& Kim, 1998). Para remediar estas falencias en las pruebas ADF, PP y KPSS se realizan las pruebas de t mínimo desarrollada por Zivot y Andrews y la prueba de Perron (Zivot \& Andrews, 1992; Perron, 1997). Estas son pruebas de raíz unitaria que permiten la estimación endógena del punto de quiebre $\left(T B_{k}\right)$. Se prueba la hipótesis nula de raíz unitaria sin cambio estructural frente a la alternativa de presencia de cambio estructural, lo cual incluye la posibilidad de raíz unitaria con quiebres estructurales (Rodríguez, 2009). Al mismo tiempo, esta prueba identifica endógenamente el punto del cambio estructural más significativo en la serie de tiempo examinada (Waheed, et al., 2006). Los resultados de estas pruebas se presentan en las tablas 2 y 3 , respectivamente.

Tabla 2. Pruebas de raíces unitarias con cambio estructural Zivot y Andrews (1992)

\begin{tabular}{cccc}
\hline \multirow{2}{*}{ Variable } & \multicolumn{3}{c}{ Modelo } \\
\cline { 2 - 4 } & $\mathrm{A}$ & $\mathrm{B}$ & $\mathrm{C}$ \\
\hline $\mathrm{cg}_{\mathrm{t}}$ & $-5,625^{* * *}$ & $-5,388^{* * *}$ & $-4,876^{* *}$ \\
Fecha de cambio & 1988 & 1990 & 1995
\end{tabular}




\begin{tabular}{cccc}
\hline \multirow{2}{*}{ Variable } & \multicolumn{3}{c}{ Modelo } \\
\cline { 2 - 4 } & A & B & C \\
\hline$y_{\mathrm{t}}$ & $-4,652$ & $-4,728$ & $-4,368$ \\
Fecha de cambio & 1986 & 1986 & 1987 \\
prg $_{\mathrm{t}}$ & $-5,205^{* *}$ & $-5,079^{* *}$ & $-4,652^{* *}$ \\
Fecha de cambio & 1989 & 1989 & 1986 \\
\hline
\end{tabular}

Nota: $\left(^{*}\right)$ indica significancia al 10\%, $\left(^{* *}\right)$ indica significancia al 5\% y $\left(^{* *}\right)$ indica significancia al 1\%. El Modelo A: Constante y Tendencia; Modelo B: Constante y Modelo C: Tendencia. Valores críticos al 5\%: Modelo A: -5,08; Modelo B: $-4,80$ y Modelo C: $-4,42$. Valores críticos al 1\%: Modelo A: -5,57; Modelo B: $-5,34$ y Modelo C: $-4,93$. Periodo: 1980-2012.

Tabla 3. Pruebas de raíces unitarias con cambio estructural Perron (1997)

\begin{tabular}{cccc}
\hline \multirow{2}{*}{ Variables } & \multicolumn{3}{c}{ Modelos } \\
\cline { 2 - 3 } & IO1 & IO2 & AO \\
\hline cg & $-5,435^{* *}$ & $-6,011^{* *}$ & $-5,023^{* *}$ \\
Fecha de cambio & 1986 & 1999 & 1994 \\
$y_{t}$ & $-4,810$ & $-4,922$ & $-4,313$ \\
Fecha de cambio & 1984 & 1984 & 1986 \\
prg & $-11,052^{* *}$ & $-5,899^{* *}$ & $-4,177$ \\
Fecha de cambio & 1986 & 1986 & 1988 \\
\hline
\end{tabular}

Nota: $\left(^{*}\right)$ indica significancia al 10\%, $\left({ }^{* *}\right)$ indica significancia al $5 \%$ y $\left(^{* * *}\right)$ indica significancia al $1 \%$. IO1 (innovational outlier) con cambio en el intercepto; IO2 (innovational outlier) cambio en el intercepto y en la pendiente de la tendencia y AO (additive outlier) cambio instantáneo en la pendiente de la tendencia. Valores críticos al $5 \%$ : IO1: -5,23; IO2: -5,59 y AO: -4,83 (Perron, 1997). Valores críticos al 10\%: IO1: -4,92; IO2: -5,29 y AO: -4,48 (Perron, 1997). Periodo: $1980-2012$.

Los resultados presentados en las tablas 2 y 3 permiten concluir que la serie consumo de gasolina $(\mathrm{cg})$ presenta cambio estructural en los años 1986, 1988, 1990, 1994, 1995 y 1999. Los resultados de las pruebas también indican que el ingreso $(y)$ no presenta cambio estructural, mientras que el precio relativo de la gasolina (prg) sí presenta en los años 1986 y 1989.

Habiendo determinado que todas las series tienen el mismo orden de integración se especificó un modelo VAR con dos rezagos ${ }^{3}$ y se aplicó el procedimiento de Johansen. De esta manera, el VAR estimado es el siguiente:

3 El número de rezagos del VAR fue seleccionado con base en los criterios de información Akaike (AIC) y de Hannan Quinn (HQ). Los modelos VAR son, en esencia, sistemas de ecuaciones. Su formalización se presenta en el apéndice metodológico. 


$$
\begin{aligned}
& c g_{t}=\mu_{1}+\gamma_{11} c g_{t-1}+\gamma_{12} c g_{t-2}+\beta_{11} \mathrm{y}_{t-1}+\beta_{12} \mathrm{y}_{t-2}+\alpha_{11} p r g_{t-1}+\alpha_{12} p r g_{t-1}+u_{1 t} \\
& y_{t}=\mu_{2}+\gamma_{21} c g_{t-1}+\gamma_{22} c g_{t-2}+\beta_{21} \mathrm{y}_{t-1}+\beta_{22} \mathrm{y}_{t-2}+\alpha_{21} p r g_{t-1}+\alpha_{22} p r g_{t-2}+u_{2 t} \\
& \operatorname{prg}_{t}=\mu_{3}+\gamma_{31} c g_{t-1}+\gamma_{32} c g_{t-2}+\beta_{31} \mathrm{y}_{t-1}+\beta_{32} \mathrm{y}_{t-2}+\alpha_{31} p r g_{t-1}+\alpha_{32} p r g_{t-2}+u_{3 t}
\end{aligned}
$$

Con la prueba de cointegración se pretende demostrar que hay al menos una relación de equilibrio estable de largo plazo entre las variables incluidas en el modelo y con ello descartar la posibilidad de proponer una relación espuria (Granger y Newbold, 1974; Hendry, 1980). El procedimiento de Johansen es más poderoso en la prueba de cointegración que el propuesto por Engle y Granger, debido a que no plantea una elección a priori entre variables endógenas y exógenas y además porque estima con precisión el número de relaciones de cointegración, a la vez que permite encontrar (dentro de la misma estimación) la ecuación de corrección de error. Las pruebas de la traza y la raíz característica máxima permiten determinar el número de relaciones de cointegración (Johansen \& Juselius, 1990). La hipótesis nula en la primera de estas pruebas es que el número de vectores de cointegración es menor o igual a $r$, mientras que en la segunda prueba la hipótesis nula es que el rango de la matriz $\Pi$ de coeficientes de las variables endógenas es igual a $r$ (Asteriou y Hall, 2006).

Los resultados de la prueba de la traza y de la raíz característica máxima presentados en la tabla 4 permiten determinar la existencia de un vector de cointegración para el modelo especificado (ecuación 2); por lo que es posible identificar que existe una relación de equilibrio de largo plazo entre las varia-

\begin{tabular}{|c|c|c|c|c|}
\hline \multirow{2}{*}{ Ho } & \multirow{2}{*}{ Traza } & Valores críticos & \multirow{2}{*}{$\lambda$-max } & \multirow{2}{*}{$\begin{array}{c}\text { Valores críticos } \\
5 \%\end{array}$} \\
\hline & & $5 \%$ & & \\
\hline$r=0$ & $29,92^{*}$ & 24,27 & $20,18^{*}$ & 17,79 \\
\hline $\mathrm{r} \leq 1$ & 9,73 & 12,32 & 8,97 & 11,22 \\
\hline $\mathrm{r} \leq 2$ & 0,75 & 4,12 & 0,76 & 4,13 \\
\hline
\end{tabular}
bles consideradas.

Tabla 4. Prueba de cointegración basada en el procedimiento de Johansen

Nota: ${ }^{*}$ Indica el rechazo de la hipótesis nula al 5\% de significancia. Traza: prueba de la traza. $\lambda$-max: prueba de la raíz característica máxima. $r$ : es el número de vectores de cointegración. Número de rezagos en el VAR: 2. El modelo VAR incluye la variable sin restringir d85 (dummy de pulso en 1985). Periodo: 1980-2012.

Normalizando el vector de cointegración, asociada a la raíz característica máxima, como una ecuación de demanda de gasolina se pueden obtener los coeficientes de largo plazo, como se muestra en la tabla 5. Los coeficientes esti- 
mados son todos significativos y los signos que arrojan están de acuerdo con lo señalado por la teoría económica y la evidencia internacional.

Tabla 5. Ecuación normalizada: $c g_{t}=\beta_{0}+\beta_{1} y_{t}+\beta_{2} \operatorname{prg}_{t}+\varepsilon_{t}$

\begin{tabular}{ccc}
\hline & $\beta_{1}$ & $\beta_{1}$ \\
\hline$c g_{t}$ & $0,673^{* * *}$ & $-0,256^{* *}$ \\
& $(0,001)$ & $(0,104)$ \\
\hline
\end{tabular}

Notas: ${ }^{* * *},{ }^{* *} \mathrm{y} *$ significa que el coeficiente es significativo al $1 \%, 5 \%$ y $10 \%$ respectivamente. Los números entre paréntesis son los errores estándar. Log likelihood: 190,1 Periodo: 1980-2012.

De acuerdo con estos resultados, y conforme se ha evidenciado en la de literatura internacional, el consumo de gasolina en la ZMVM guarda una relación positiva con el nivel de ingresos y negativa con el aumento de los precios de la gasolina. Tanto la elasticidad ingreso como la elasticidad precio son menores que uno; sin embargo, la demanda de gasolina en la ZMVM es más sensible a cambios en el ingreso que a cambios en los precios. De esta manera, un aumento de $1 \%$ en el precio de la gasolina estaría asociado con una disminución del consumo de gasolina de $0,25 \%$, mientras que un aumento del mismo nivel en el ingreso estaría acompañado de un aumento del consumo de 0,67\%.

Los resultados obtenidos en este trabajo son consistentes con la amplia literatura que existe en el ámbito internacional tanto para países de América Latina como para países desarrollados (Dahl y Sterner, 1991; Espey, 1998). En Brasil, Nappo (2007) estimó una función de demanda de gasolina utilizando la técnica de cointegración, encontrando que la elasticidad ingreso es de 0,68 . Para el caso de Perú, Vásquez (2005) encontró que la sensibilidad de la demanda de gasolina ante aumentos de su precio es de 0,64. Para 18 países de la OCDE, Baltagi y Griffin (1983) estimaron que la elasticidad ingreso de la demanda de gasolina es de 0,66. En el ámbito nacional en Brasil y México la elasticidad precio de la demanda de gasolina ha sido estimada en $-0,20$ y $-0,28$ respectivamente (Reyes, 2010; Nappo, 2007). Al evaluar en Los Ángeles y Connecticut, la efectividad de establecer un impuesto a la gasolina como política para reducir la contaminación del aire, se encontró que la elasticidad precio se encuentra entre - 0,5 y -0,7 (Sipes y Mendelsohn, 2001).

Las pruebas de causalidad de Granger (1969) presentadas en la tabla 6 evidencian que existe una causalidad entre cambios en el Producto Interno Bruto y el consumo de gasolina, cambios en el precio de la gasolina y el consumo de esta y entre cambios en el precio de la gasolina y el Producto Interno Bruto. Esto indica la presencia de relación entre los cambios en las variables incluidas en el modelo, las cuales pueden considerarse en el diseño de la política pública para controlar el consumo de la gasolina en la zona de estudio. De este modo, puede 
utilizarse el modelo econométrico para explicar, pronosticar y simular diversos escenarios de política económica.

Tabla 6. Prueba de Causalidad de Granger

\begin{tabular}{lccc}
\hline \multicolumn{1}{c}{ Hipótesis nula } & Observaciones & Estadístico F & Prob. \\
\hline PIB no causa el consumo de gasolina & 31 & 0,14538 & 0,7058 \\
Consumo de gasolina no casusa el PIB & & $\mathbf{3 , 8 1 4 0 8}$ & $\mathbf{0 , 0 6 0 5}$ \\
Precio de la gasolina no causa el consumo de gasolina & 31 & 0,11973 & 0,7318 \\
Consumo de gasolina no causa el precio de la gasolina & & $\mathbf{7 , 1 1 5 5 8}$ & $\mathbf{0 , 0 1 2 4}$ \\
Precio de la gasolina no causa el PIB & 31 & 0,01054 & 0,9189 \\
PIB no causa el precio de la gasolina & & $\mathbf{5 , 2 8 2 8 2}$ & $\mathbf{0 , 0 2 8 9}$ \\
\hline
\end{tabular}

Nota: Los valores en negritas indican el rechazo de la hipótesis nula. Número de rezagos: 2. Periodo: 1980-2012.

Para encontrar los efectos de corto plazo del consumo de gasolina se estimó un modelo de corrección de errores. De acuerdo con esta metodología, se debe especificar un VAR en diferencia que contenga los residuos generados en la regresión de cointegración de la tabla 5 con rezago y debe pasar todas las pruebas de correcta especificación, de manera que permita realizar pronóstico con alto grado de confiabilidad (Loria, 2007). Se siguió el procedimiento de lo general a lo particular de Hendry (1995) y de acuerdo con el grado de significancia estadística se fueron eliminando variables. Los resultados del modelo de corrección de errores se presentan en la tabla 7 .

Tabla 7. Resultado del Modelo de Corrección de Errores

\begin{tabular}{cc}
\hline Variable & $\Delta c g$ \\
\hline$\Delta y_{t}$ & $0,496(0,083)^{* * * *}$ \\
$\Delta p r g_{t}$ & $-0,126(0,028)^{* * * *}$ \\
$u_{t-1}$ & $-0,248(0,044)^{* * * *}$ \\
D85 & $0,093(0,013)^{* * * *}$ \\
$\mathrm{R}^{2}$ & 0,844 \\
Normalidad JB & $\chi^{2}: 3,273[0,194]$ \\
Autocorrelación LM (2 rezagos) & $F: 1,325[0,283]$ \\
Heteroscedasticidad ARCH (2 rezagos) & $F: 0,036[0,963]$ \\
\hline
\end{tabular}

Nota: Los números entre paréntesis de los coeficientes son el error estándar. * indica significancia estadística al $10 \%$; ** indica significancia estadística al $5 \%$; ${ }^{* * *}$ significancia estadística al $1 \% . R^{2}$ es el coeficiente de determinación. JB es el estadístico Jarque Bera, utilizado para probar normalidad. LM es el Multiplicador de Lagrange, utilizado para probar autocorrelación. ARCH es la heterocedasticidad condicional autoregresiva para la prueba de heterocedasticidad. Periodo: 1980-2012. 


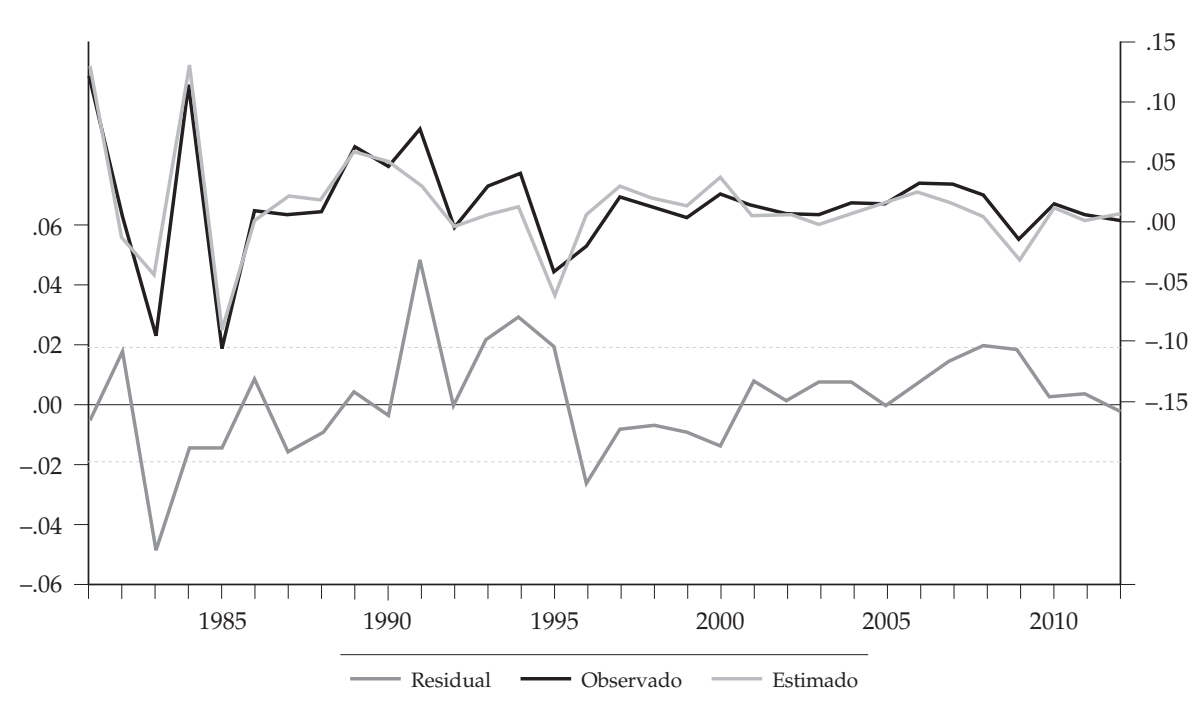

Figura 4. Valores observados, estimados y residuales del consumo de gasolina en la ZMVM

Fuente: Elaborado por los autores con base en las estimaciones.

Los resultados de la tabla 7 representan una buena aproximación del proceso generador de infamación (Spanos, 1986) y permiten ver que el modelo cumple con las pruebas de diagnóstico, es decir, los errores se distribuyen como una normal, no presentan problemas de autocorrelación ni de heteroscedasticidad. La figura 4 permite ver que el modelo reproduce de buena manera la evolución de los datos y por consiguiente los resultados pueden ser utilizados para realizar pronósticos al emplear escenarios diferentes en las variables exógenas. Los resultados obtenidos indican una elasticidad ingreso de corto plazo de 0,49 y una elasticidad precio de corto plazo de - 0,12 , las cuales son consistentes con la literatura internacional (Bentzen, 1994; Eltony, 1996; Eltony y AI-Mutairi, 1995; Hughes, et al., 2006; Johnston y DiNardo, 1997; Ramanathan, 1999; Sterner, et al., 1992). En la tabla 8 se presenta, de manera resumida, la magnitud de las elasticidades de largo y corto plazo de la demanda de gasolina para la ZMVM.

Tabla 8. Elasticidades de largo y corto plazo de la demanda de gasolina

\begin{tabular}{ccc}
\hline Elasticidad & Largo plazo & Corto plazo \\
\hline Ingreso & 0,67 & 0,49 \\
Precio & $-0,25$ & $-0,12$ \\
\hline
\end{tabular}

Fuente: Elaboración propia con base en los resultados de las estimaciones (tablas 5 y 6). 


\section{Trayectoria del consumo de gasolina en la ZMVM}

Utilizando los resultados del modelo de corrección de errores se realizaron proyecciones para el consumo de gasolina que permiten definir el escenario base en un horizonte de tiempo definido hasta 2020. Este escenario base se interpreta como la trayectoria que tomaría el consumo de gasolina en la ZMVM si no se implementa ninguna política pública para reducir la demanda del combustible, lo que es conocido en la literatura como business as usual (BAU). De igual manera, se construyeron escenarios alternativos que permiten comparar los resultados de diferentes políticas para reducir el consumo de gasolina. Los supuestos bajo los cuales se construyen estos escenarios se presentan en la tabla 9.

Tabla 9. Escenarios contemplados en la simulación de la trayectoria de la demanda de gasolina a 2020 (Tasas de crecimiento en porcentaje)

\begin{tabular}{ccc}
\hline Escenario & PIB & Precio \\
\hline Escenario Base & 3,5 & - \\
\hline Pesimista & 3,5 & 2,0 \\
\hline Moderado & 3,5 & 3,0 \\
\hline Optimista & 3,5 & 5,0 \\
\hline
\end{tabular}

Nota: (--) Indica que la variable no registra ningún cambio entre 2013 y 2020.

Los resultados de la simulación, con los supuestos considerados en cada escenario, permiten definir la trayectoria del consumo de gasolina hasta 2020 en la ZMVM. De esta manera, se encuentra que sin la implementación de políticas se estarían consumiendo 8966 millones de litros de gasolina en 2020, lo que representa 1228 millones de litros más que en 2012. Esto indica que de presentarse las condiciones mencionadas, el consumo de gasolina crecería a una tasa promedio anual de 1,85\% entre 1912 y 2020. La utilidad de definir la línea base es que permite realizar comparaciones con cualquier medida de política pública que se pretenda implementar para reducir el consumo de gasolina. Por consiguiente, con esta línea base se pueden comparar los resultados que se obtendrían con una política como la reducción del subsidio a la gasolina.

Como es normal, en trabajos que involucran variables económicas, la trayectoria proyectada para el consumo de gasolina trae asociada un grado de incertidumbre que tiene que ver con los posibles valores que pueden tomar en un futuro las variables exógenas del modelo. Con el fin de incorporar esta incertidumbre en los resultados se presenta en la figura $5 \mathrm{el}$ Fan Chart de la trayectoria del consumo de gasolina en los escenarios base y alternativos en la ZMVM. El Fan Chart representa la función de probabilidad de los valores futuros de una 
variable, condicional a la información conocida en el presente, con la ventaja, frente a otras formas de presentar pronósticos, de describir completamente la densidad marginal de pronóstico en cada uno de los periodos del horizonte de tiempo y, además, su formulación permite que la densidad marginal de pronóstico sea asimétrica. Estas ventajas hacen al Fan Chart más deseable para presentar los riesgos de que se cumplan las metas sobre el valor futuro de nuestra variable de interés (julio, 2006).

(a) Escenario Base

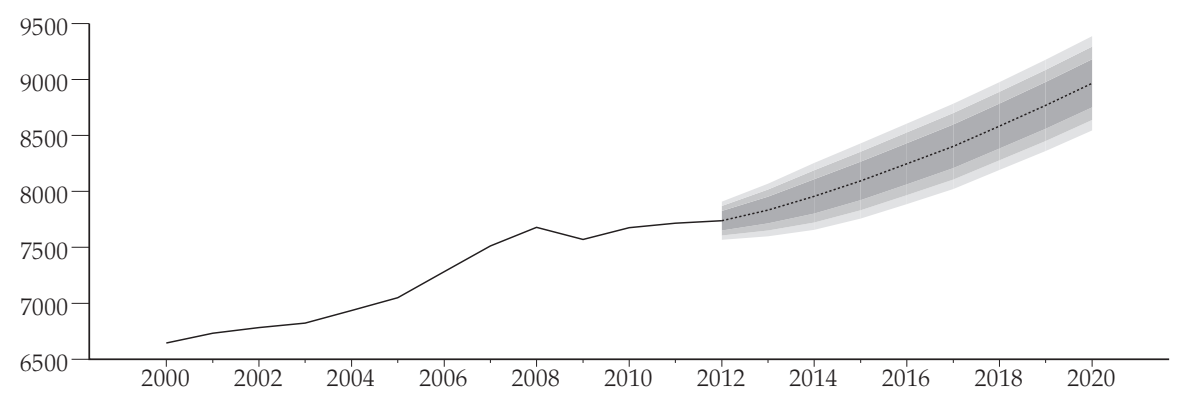

(b) Escenario pesimista

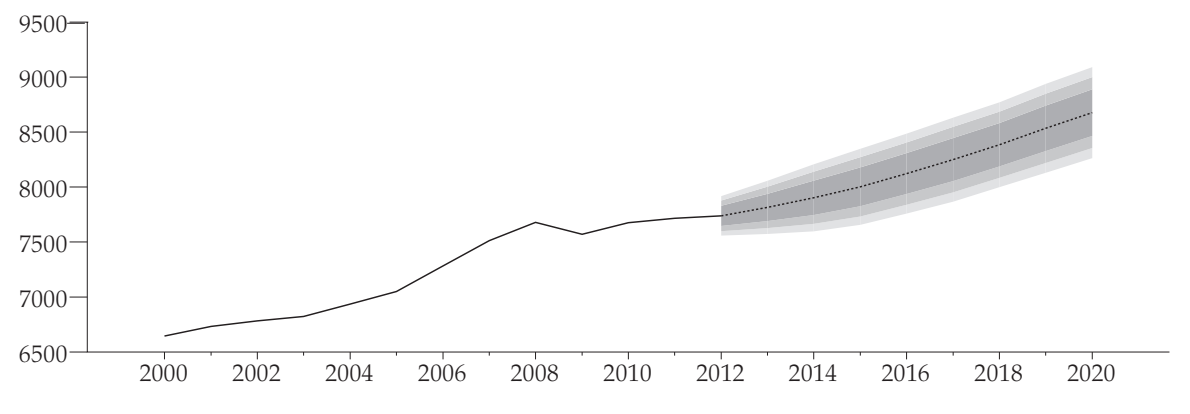

(c) Escenario moderado

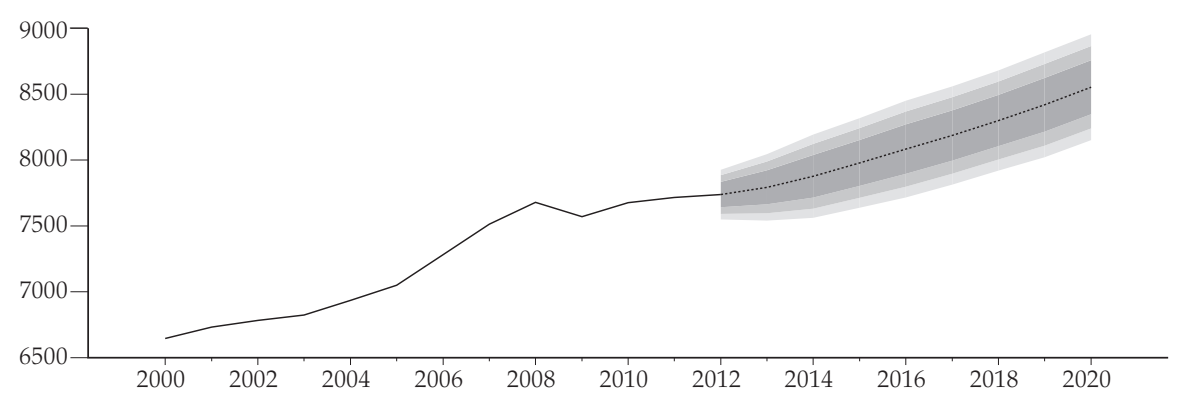


(d) Escenario optimista

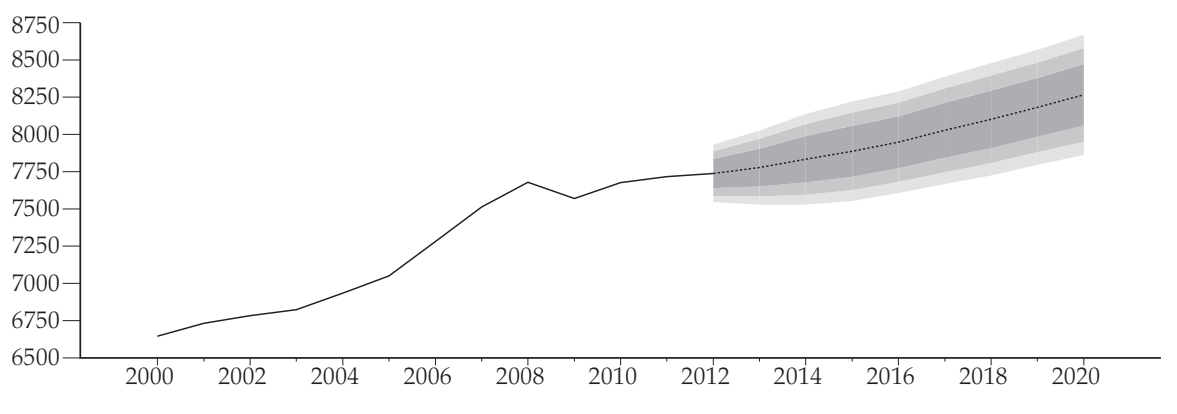

Figura 5. Trayectorias del consumo de gasolina en la ZMVM en el BAU y escenarios alternativos

Fuente: Elaborado por los autores con base en resultado de las simulaciones.

Nota: La trayectoria del consumo de gasolina está en millones de litros.

La intensidad de las sendas representadas en la figura 5 define el grado de probabilidad con que el consumo de gasolina podría alcanzar los valores pronosticados. De esta manera, en el escenario base, con un $80 \%$ de probabilidad, el consumo de gasolina en la ZMVM crecerá a una tasa promedio anual de entre $1,6 \%$ y 2,1 \% para el periodo 2012-2020. Esto indica que el consumo de gasolina se encontrará en 2020 en un rango entre 8637 y 9294 millones de litros (panel a de la figura 5); es decir, se estarían consumiendo entre 1032 y 1423 millones de litros más que en 2012. Al incorporar los supuestos del escenario pesimista se obtiene una nueva senda de consumo con horizonte a 2020 (panel b de la figura 5), en la que el consumo de gasolina en la ZMVM crece, con un $80 \%$ de probabilidad, a una tasa promedio anual de entre 1,2\% y 1,7\% para el periodo 2012-2020, lo que indica que el consumo de gasolina se encontrará en 2020 en un rango entre 8354 y 8999 millones de litros, esto es, se estará consumiendo entre 755 y 1122 millones de litros más que en 2012. Desde luego esta trayectoria arroja un menor consumo de gasolina que el escenario base.

El segundo escenario alternativo (moderado) incluye como supuesto un crecimiento del precio del 3\% cada año y un crecimiento del PIB del 3,5\%. Al incorporar los supuestos al modelo de gasolina se obtiene una tercera senda de consumo con horizonte a 2020 (panel c de la figura 5), según la cual el consumo de gasolina en la ZMVM crecerá, con $80 \%$ de probabilidad, a una tasa promedio anual entre 1,03\% y 1,48\% para el periodo 2012-2020. Esto es, el consumo de gasolina para 2020 estará entre 8239 y 8865 millones de litros de gasolina, con lo cual se estarían consumiendo entre 648 y 980 millones de litros más que en 2012.

El escenario optimista para el consumo de gasolina considera un aumento mayor del precio de la gasolina y el crecimiento de 3,5\% del PIB, con lo que se 
obtiene cuarta senda de consumo de gasolina con horizonte a 2020 (panel d, de la figura 5). En este escenario, el consumo de gasolina en la ZMVM crece, con $80 \%$ de probabilidad, a una tasa promedio anual entre $0,59 \%$ y $1,06 \%$ para el periodo 2012-2020, lo que llevaría a que el consumo de gasolina se encuentre en 2020 en un rango entre 7951 y 8580 millones de litros, es decir que se estarían consumiendo entre 363 y 692 millones de litros más que en 2012. Evidentemente, con los supuestos de este escenario, la trayectoria arroja un menor consumo de gasolina que el escenario base y los escenarios alternativos pesimista y moderado.

\section{Consideraciones finales e implicaciones de política}

El estudio de la demanda de gasolina para la ZMVM resulta de mucho interés por los efectos económicos, ambientales y sociales. Al ser esta zona metropolitana una de las más contaminadas del mundo, el análisis del sector transporte y, de manera particular, de la demanda de gasolina, se convierte en un tema sobre el que pueden extraerse oportunidades de política pública. Los resultados del modelo econométrico estimado muestran que la elasticidad ingreso de la demanda de combustible es mayor que la elasticidad precio, por lo que una política pública que busque reducir significativamente el consumo de gasolina no puede basarse únicamente en incentivos de precios. Esto es, la reducción del consumo de gasolina que se conseguiría por el aumento del precio sería contrarrestada por la mayor demanda que se deriva del aumento del ingreso.

Esta relación entre las magnitudes de las elasticidades precio e ingreso tiene implicaciones económicas y ambientales. Por una parte, la baja elasticidad precio de la demanda puede generar mayores ingresos fiscales vía recaudación de los impuestos asociados al consumo de gasolina, sumado a las transferencias evitadas por el desmonte de los subsidios al combustible. No obstante, el mayor consumo de gasolina también está asociado con el aumento de emisiones de gases de efecto invernadero y contaminantes locales que tienen efectos sobre la salud de la población. Ello implica que al lograrse disminuir el consumo de combustibles, se estarían obteniendo ganancias ambientales y co-beneficios en materia de salud.

De esta manera, para alcanzar reducciones importantes en el consumo de gasolina se requieren políticas públicas complementarias a la eliminación del subsidio, como las normas que regulan el rendimiento de los combustibles, incentivos económicos dirigidos a reducir la venta de autos. En este mismo sentido, deben priorizarse políticas orientadas a mejorar el transporte público colectivo, con infraestructura sustentable, como el metrobus, metro y trolebus. Este tipo de transporte público no solo tiene la capacidad para transportar mayor 
cantidad de pasajeros por litro de gasolina consumido, sino que, si se logra que funcione de manera eficiente, permitiría un cambio modal de vehículos particulares hacia el transporte público, lo que implica reducciones adicionales de consumo de combustibles y disminuciones en la congestión vehicular.

Como es muy probable que el desmonte de los subsidios tenga efectos de segunda vuelta sobre los deciles más bajos, representados en aumentos de los precios de alimentos y del transporte público, se requiere también que la eliminación del subsidio venga acompañada de políticas de compensación focalizadas hacia la población más pobre.

Bajo los escenarios considerados en la construcción de las trayectorias del consumo de gasolina, incluso en el escenario más optimista, es evidente que en los próximos años aumentará el consumo de gasolina como resultado, principalmente, del mayor ingreso del que dispondrán las familias y la baja respuesta de los usuarios de los autos al aumento del precio del combustible. En este contexto, iniciativas dirigidas a desincentivar el uso de los autos particulares, como el aumento del costo de los estacionamientos y de la circulación, pueden contribuir también al objetivo de reducir el consumo de gasolina y a internalizar los costos de los daños causados.

\section{Apéndice metodológico}

A pesar de las diferentes metodologías utilizadas en la literatura para modelar la demanda de combustibles, la econometría moderna ha encontrado consenso en que el enfoque de cointegración ofrece ventajas en el tratamiento de los datos que pueden mostrar alguna tendencia en el periodo de estudio (Bentzen, 1994; Crôtte, et al., 2010; Galindo, 2005, 2008; Eltony y AI-Mutairi, 1995). Para datos estocásticos, es decir, si la tendencia de algunos periodos es diferente de la tendencia de otros, la técnica de cointegración evita el problema de regresión espuria (Crôtte, et al., 2010; Enders, 2004; Granger, 1981). Por medio de esta metodología se puede comprobar empíricamente la existencia de una combinación de las variables que presentan una relación estable en el tiempo y por consiguiente identificar una relación de equilibrio. En este sentido, los modelos considerados en este trabajo se basan en el análisis de cointegración y modelos de corrección de error (MCE), los cuales toman en cuenta las características de los datos de serie de tiempo.

Las principales ventajas de usar este modelo tienen que ver con la facilidad para distinguir entre las respuestas de corto y largo plazo y, al mismo tiempo, con que la velocidad de ajuste hacia los valores de largo plazo pueden ser estimados directamente. La estimación de la demanda de gasolina 
utilizando la técnica de cointegración consta de tres pasos (Crôtte, et al., 2010; Eltony, 1996).

- El primer paso es examinar las series de tiempo que hacen parte del modelo, con el fin de determinar si tienen raíz unitaria, es decir, si su primera o segunda diferencia, o si la n-diferencia de la serie es estacionaria.

- Si se encontró que las variables no son estacionarias, entonces el segundo paso es investigar la cointegración entre las variables. Si se comprueba que están cointegradas, es decir, las variables poseen una relación de largo plazo, entonces las elasticidades de largo plazo pueden estimarse a partir de la regresión de cointegración.

- El tercer paso es encontrar las elasticidades de corto plazo y la velocidad de ajuste a partir de un modelo de corrección de errores.

De acuerdo con estos pasos, es necesario realizar pruebas de raíz unitaria para identificar el orden de integración de las series. Existen varias pruebas de raíz unitaria entre las que se destacan las pruebas Dickey - Fuller Aumentada, Phillips y Perron, y la prueba Kwiatkowsky, Phillips, Schmidt y Shin (Phillips y Perron, 1988). La prueba Dickey - Fuller Aumentada consiste en correr una regresión para cada serie considerada en el modelo, con la primera diferencia como variable dependiente y las variables en niveles y, en primera diferencia rezagada, como variables explicativas (Asteriou \& Hall, 2006; Enders, 2004; Lutkepohl \& Kratzig, 2004). La prueba ADF formalmente se puede plantear como:

$$
\Delta X_{t}=\beta+\alpha X_{t-1}+\sum_{i=1}^{n} \phi_{1} \Delta X_{t-i}+e_{t}
$$

Donde $\Delta$ es el operador primera diferencia y $e_{t}$ es el error aleatorio estacionario. Las hipótesis de esta prueba se plantean sobre el parámetro $\alpha$, el cual define si la serie es o no estacionaria.

$H_{0}: \alpha=0 \rightarrow$ Existe raíz unitaria. La serie no es estacionaria.

$H_{0}: \alpha \neq 0$ y $\alpha<0 \rightarrow$ No existe raíz unitaria. La serie es estacionaria.

Dado que las series deben ser estacionarias, entonces nos interesa rechazar la hipótesis nula, lo que ocurre cuando el coeficiente del término en niveles rezagado $(\alpha)$ es estadísticamente significativo y mayor en valor absoluto que los valores críticos reportados en tabla. 
Con el fin de encontrar una relación que represente adecuadamente la dinámica del consumo de gasolina es necesario hallar una ecuación de equilibrio de largo plazo entre las variables del modelo. El procedimiento de Johansen supera las deficiencias que presenta la metodología de Engle-Granger, convirtiéndose en un método apropiado y ampliamente utilizado para identificar las relaciones de cointegración (Enders, 2004; Johansen, 1988; Engle y Granger, 1987). El concepto de cointegración es la noción estadística equivalente a la idea de equilibrio estable, dado que cuando existe una relación de este tipo entre variables económicas, las desviaciones de esta relación no pueden ser fuertes ni crecer ilimitadamente; de esta forma, la cointegración de las variables de un modelo da validez al mismo a largo plazo (Pérez, 2006). Este método se basa en estimar un modelo VAR, como se especifica en la ecuación 7 (Asteriou y Hall, 2006).

$$
Z_{t}=A_{1} Z_{t-1}+A_{z} Z_{t-1}+\ldots+A_{k} Z_{t-k}+u_{t}
$$

Donde $Z_{t}$ es una matriz que incluye a las variables endógenas y $U_{t}$ es un vector de errores. Sumando $Z_{t-1}, \ldots, Z_{t-k}$ y $A_{1} Z_{t-2}, A_{2} Z_{t-3}, \ldots, A_{k-1} Z_{t-k}$ en ambos lados de la ecuación (7), esta puede ser reformulada en forma de un modelo de corrección de errores (ECM) como se prueba en (Johansen, 1988).

$$
\Delta Z_{t}=\Gamma_{1} \Delta Z_{t-1}+\Gamma_{2} \Delta Z_{t-2}+\ldots+\Gamma_{\mathrm{k}-1} \Delta Z_{t-\mathrm{k}-1}+\prod Z_{t-1}+u_{t}
$$

Donde $\Gamma_{i}=\left(I-A_{1}-A_{2}-\ldots-A_{k}\right)(i=1,2, \ldots, k-1)$ y $\prod_{i}=-\left(I-A_{1}-A_{2}-\ldots-A_{k}\right)$. Tanto en la ecuación (7) como en (8), la matriz $\prod$ de coeficientes de las variables endógenas es idéntica y refleja el ajuste dinámico permanente de las primeras diferencias de las variables respecto de sus niveles (Charemza \& Deadman, 1999). El teorema de representación de Granger indica que si existe cointegración existe necesariamente una representación de la relación de largo plazo en una de corto plazo que corrige el error que le es consustancial y evita que las series cointegradas se dispersen en el tiempo (Loria, 2007). Este mismo teorema plantea que la cointegración es una condición necesaria para que haya modelos de corrección de errores, los cuales encuentran relaciones estables de largo plazo y el juste estadístico del desequilibrio que puede existir en el corto plazo.

Respecto del rango de la matriz $\prod$, es decir, el número de relaciones linealmente independientes y estacionarias, es necesario precisar que si este es igual a $n$ (número de variables explicativas incluidas en el VAR), el vector $Z_{t}$ es estacionario, lo que implica que las variables incluidas en el modelo son I(0)y se pueden estimar mediante mínimos cuadrados ordinarios. Si el rango de $\Pi$ es menor que $n$, entonces existe una representación de $\Pi$ tal que $\Pi=\alpha \beta^{\prime}$ donde $\alpha$ 
representa el parámetro de velocidad del ajuste al equilibrio y $\beta^{\prime}$ es la matriz de cointegración que tiene la siguiente propiedad: $\beta^{\prime} Z_{t} \sim I(0)$, donde $Z_{t} \sim I$ (1). Esto quiere decir que las variables contenidas en $Z_{t}$ se cointegran por medio de los vectores $\beta_{1}, \beta_{2}, \ldots \beta_{n^{\prime}}$ que son las columnas de la matriz de cointegración (Loria, 2007). En la práctica el rango de la matriz $\prod$ es estimado en el procedimiento de Johansen mediante el método de máxima verosimilitud. Este procedimiento utiliza los estadísticos de la traza y el de la raíz característica máxima.

\section{Referencias}

Akinboade, O. A., Ziramba, E. y Kumo, W. (2008). The Demand for Gasoline in South Africa: An Empirical Analysis Using Co-Integration Techniques. Energy Economics, 30(6), 3222-3229.

Alves, D. y Bueno, R. (2003). Short-Run, Long-Run and Cross Elasticities of Gasoline Demand in Brazil. Energy Economics, 25, 191-199.

Amengual, D. y Cubas, G. (2002). Imposición óptima a las naftas y el gasoil: Un análisis empírico para Uruguay (1988-2001). Recuperado de http:/ /www. bcu.gub.uy/autoriza/peiees/jor/2002/iees03j370702.pdf.

Archibald, R. y Gillingham, R., (1980). An Analysis of the Short-Run Consumer Demand for Gasoline Using Household Survey Data. The Review of Economics and Statistics, 62(4), 623-629.

Asteriou, D. y Hall, S. (2006). Applied Econometrics. A Modern Approach Using Eviews and Microfit. Revised edition. New York: Palgrave Macmillan.

Baltagi, B. H. y Griffin, J. M. (1983). Gasoline Demand in the OECD. An Application of Pooling and Testing Procedures. European Economic Review, 22, 117-137.

Becker, G. S., (1965). A Theory of the Allocation of Time. Economic Journal, 75, 493-517.

Belhaj, M. (2002). Vehicle and Fuel Demand in Morocco. Energy Policy, 30, 11631171.

Bentzen, J. (1994). An Empirical Analysis of Gasoline Demand in Denmark Using Co Integration Techniques. Energy Economics, 16, 139-143.

Birol, F. y Guerer, N. (1993). Modelling the Transport Sector Fuel Demand for Developing Economies. Energy Policy, 1163-1172.

Charemza, W. y Deadman, D. (1999). New Directions in Econometric Practice. General to Specific Modelling, Cointegration and Vector Autoregression. $2^{a}$ edition. Reino Unido: Edward Elgar Publishing.

Crotte Alvarado, A. (2008). Estimation of Transport Related Demand Elasticities in Mexico City. An Application to Road User Charging. Centre for Transport 
Studies. Recuperado de http://www.cts.cv.ic.ac.uk/documents/theses/ CrottePhD.pdf

Crôtte, A., Noland, R. y Graham, D. (2010). An Analysis of Gasoline Demand Elasticities at the National and Local Levels in Mexico. Energy Policy, 38, 4445-4456.

Dahl, C. y Sterner, T. (1990). The Pricing of and the Demand for Gasoline: a Survey of Models. Memorandum 132.

Dahl, C. y Sterner, T. (1991). Analysing Gasoline Demand Elasticities: A Survey. Energy Economics, 13(3), 203-210.

Deaton, A. y Muellbauer, J. (1980), Economics and Consumer Behavior. New York: Cambridge University Press.

Dickey, D. A. y Fuller, W. A. (1981). Likelihood Ratio Statistics for Autoregressive Time Series with a Unit Root. Econometrica, 49, 1057-1072.

Eltony, M. N. y AI-Mutairi, N. H. (1995). Demand for Gasoline in Kuwait. An Empirical Analysis Using Cointegration Techniques. Energy Economics, 17(3), 249-253.

Eltony, M. N. (1996). Demand for Gasoline in the GCC: an Application of Pooling and Testing Procedures. Energy Economics, 18, 203-209.

Enders, W. (2004). Applied Econometric Time Series. Second Edition. New Jersey, Wiley.

Engle, R. F. y Granger, C. W. (Marzo, 1987). Co-Integration and Error Correction: Representation, Estimation and Testing. Econométrica, 55(2) 251-276.

Eskeland, G. S. y Feyzioglu, T. N. (1997). Is Demand for Polluting Goods Manageable? An Econometric Study of Car Ownership and Use in Mexico. Journal of Development Economics, 53, 423-445.

Espey, M. N. (1998). Gasoline Demand Revisited: an International Meta-Analysis of Elasticities. Energy Economics, 20, 273-295.

Galindo, L. M. (2005). Short- and Long-Run Demand for Energy in Mexico: A Cointegration Approach, Energy Policy, 33(9), 1179-1185.

Galindo, L. M. (2008). Estudio sobre la instrumentación de medidas de eficiencia energética y uso de biocombustibles en el sector transporte y su impacto en la calidad del aire en México. Informe Final. Convenio No. INE/A1-004/2008.,. Ciudad de México: Facultad de Economía - UNAM - INE -SEMARNAT.

Galindo, L. M. y Salinas, E. (1997). La demanda de gasolinas en México. La condición de exogeneidad y el comportamiento de los agentes económicos. México: Instituto Nacional de Ecología.

Graham, D. y Glaister, S. (2002). Review of Income and Price Elasticities of Demand for Road Traffic. London: Centre for Transportation Studies.

Granger, C. W. J. (1969). Investigating Causal Relations by Econometric Models and Cross-spectral Methods. Econometrica, 37(3), 424-438. 
Granger, C. W. J. (1981). Some Properties of Time Series Data and their Use in Econometric Model Specification, Journal of Econometrics, 16, 121-130.

Granger, C. W. J. y Newbold, P. (1974). Spurious Regressions in Econometrics. Journal of Econometrics, 2, 111-120.

Hendry, D. F. (1993). Econometrics: Alchemy or Science? Oxford: Blackwell Publishers, and Oxford University Press.

Hendry, D. F. (1995). Dynamic Econometrics. New York: Oxford University Press. Hughes, J., Knittel, C. y Sperling, D. (2006). Evidence of a Shift in the ShortRun Price Elasticity of Gasoline Demand. National Bureau of Economic Research. Working Paper Series. 12530. Recuperado de http:/ / www.nber. org/papers/w12530.

Johansen, S. (1988). Statistical Analysis of Cointegration Vectors. Journal of Economics Dinamyc and Control, 12, 231-254.

Johansen, S. y Juselius, K. (1990). Maximum Likelihood Estimation and Inference on Cointegration - with Applications to the Demand for Money, Oxford Bulletin of Economics and Statistics 52, 169-210.

Johansson, O. y Schipper, L. (1997). Measuring the Long-Run Fuel Demand of Cars. Separate Estimations of Vehicle Stock, Mean Fuel Intensity, and Mean Annual Driving Distance. Journal of Transport Economics and Policy, 31, 277-292.

Johnston, J. y DiNardo, J. (1997). Econometric Methods. Irvine: McGraw-Hill International Editions.

Julio, J. M. (2006). Implementación, uso e interpretación del Fan Chart. Revista Colombiana de Estadística, 29(1), 109-131.

Kayser, H. A. (2000). Gasoline Demand and Car Choice: Estimating Gasoline Demand Using Household Information. Energy Economics, 22, 331-348.

Kwiatkowsky, D., Phillips, P., Schmidt, P. y Shin, R. (1992). Testing the Null Hypothesis of Stationary Against the Alternative of a Unit Root. Journal of Econometrics, 54, 159-178.

Lancaster, K. J. (1966). A New Approach to Consumer Theory. Journal of Political Economy 74, 132-157.

Loria, E. (2007). Econometría con aplicaciones. México: Pearson Prentice Hall.

Lutkepohl, H. y Kratzig, M. (2004). Applied Time Series Econometrics. New York: Cambridge University Press.

Maddala, G. S., \& Kim, I. (1998). Unit Roots, Cointegration and Structural Change. Cambridge: Cambridge University Press.

Mannering, F. y Winston, C. (1985). Vehicle Demand and the Demand for New Car Fuel Efficiency. Rand J. Econ, 16, 215-236.

Mas-Colell, A., Whinston, M. y Green, J. R. (1995). Microeconomic Theory. New York: Oxford University Press. Inc. 
Mora, R. J. (2002). Introducción a la Teoría del Consumidor. De la preferencia a la estimación. Cali: Universidad ICESI. Feriva S.A.

Muth, R. F. (1966). Household Production and Consumer Demand Functions. Econométrica 34, 699-708.

Nappo, M. (2007). A demanda por gasolina no Brasil: Uma avaliação de suas elasticidades após a introdução dos carros bicombustível. São Paulo: Fundação Getúlio Vargas, Escola de Economia de São Paulo-Eesp.

Pérez, C. (2006). Econometría de las series temporales. Madrid: Pearson Prentice Hall.

Perron, P. (1997). Further Evidence on Breaking Trend Functions in Macroeconomic Variables. Journal of econometrics, 80, 335-385.

Phillips, P. C. B. y Perron, P. (1988). Testing for Unit Roots in Time Series regression. Biometrika, 75, 335-346.

Pincyck, R. S. y Rubinfeld, D. L. (1998). Microeconomics. Fourth edition. London: Prentice Hall Inc.

Polemis, M. L. (2006). Empirical Assessment of the Determinants of Road Energy Demand in Greece. Energy Economics, 28, 385-403.

Ramanathan, R. (1999). Short- and Long-Run Elasticities of Gasoline Demand in India: An Empirical Analysis Using Cointegration Techniques. Energy Economics, 21, 321-330.

Reyes Tépach, M. (2011). Análisis de los precios y de los subsidios a las gasolinas y el Diesel en México, 2007-2010. Servicios de Investigación y Análisis. Ciudad de México: Cámara de Diputados, Subdirección de Economía.

Reyes, M. O., Escalante, R. y Matas, A. (2010). La demanda de gasolinas en México: Efectos y alternativas ante el cambio climático. Economía: teoría y práctica, 32, 83-111.

Rodríguez, A. (2009). Pruebas de raíz unitaria con cambio estructural de Lee y Strazicich. San José: Banco Central de Costa Rica, Departamento de Investigación Económica.

SENER, Secretaría de Energía (2010). Prospectiva de petrolíferos 2010-2025. México: Gobierno Federal SENER.

Sipes, K. N. y Mendelsohn, R. (2001).The Effectiveness of Gasoline Taxation to Manage Air Pollution. Ecological Economics, 36, 299-309.

Spanos, A. (1986). Statistical Foundations of Econometric Modeling, Cambridge: Cambridge University Press.

Sterner, T., Dahl, C. y Franzén, M. (1992). Gasoline Tax Policy, Carbon Emissions and the Global Environment, Journal of Transport Economics and Policy, 26(2), 109-119.

Storchmann, K. (2005). Long-Run Gasoline Demand for Passenger Cars: the Role of Income Distribution. Energy Economics, 27, 25-58. 
Sultan, R. (2010). Short-Run and Long-Run Elasticities of Gasoline Demand in Mauritius: an ARDL Bounds Test Approach, Journal of Emerging Trends in Economics and Management Sciences (JETEMS), 1(2), 90-95.

Varian, Hal R. (1990). Intermediate Microeconomics. A Modern Approach. 5th. Edition. New York: Antoni Bosch Editor.

Vásquez, A. (2005). La demanda agregada de combustibles líquidos en el Perú. Documento de trabajo No. 12, Oficina de Estudios Económicos, Organismo Supervisor de la Inversión en Energía.

Waheed M., Alam, T. y Ghauri, S. P. (2006). Structural Breaks and UnitRoot: Evidence From Pakistani Macroeconomic Time Series, MPRA Paper, 1797, University Library of Munich, Germany.

Zivot, E. y Andrews, D. (1992). Further Evidence on the Great Crash, the Oil Price Shock and the Unit-Root Hypothesis. Journal of Business and Economic Statistics, 10, 251-270. 
